\title{
Smart Automated Sericulture Based On Image Processing Technique And Embedded System
}

\author{
Yogeshraj N S ${ }^{1}$,Thamilarasi $\mathrm{N}^{2}$,Sailaja $\mathrm{S}^{3}$,Poonguzhali $\mathrm{C}^{4}$, Kannagi $\mathrm{S}^{5}$ \\ 1. Student, M.E., Applied Electronics ${ }^{2,3,4,5}$ Assistant Professor, Department of Electronics and Communication Engineering, \\ Jaya Engineering College, Chennai
}

\begin{abstract}
Sericulture is the process of growing silkworms for the purpose of producing silk. India is the world's second-largest producer of silk. Sericulture is central to India's socioeconomic, economic, cultural, and political development. Temperature and humidity are important factors in the growth of healthy silkworms. at all stages, particularly during larval development. We're utilising the webcam to detect silkworms that aren't doing well. Image processing aids us in recognising infection, ill health, and non-identical phases of silkworms in our study. The colour change in the silkworm's body is mostly determined via image processing techniques. It denotes non-identical phases, such as black worms and swallow worms, as well as diseases caused by worms.
\end{abstract}

Keywords - Sericulture, Silkworm, Temperature, Humidity, Arduino, Image processing techniques(CNN).

\section{INTRODUCTION}

Because farmers are having so many problems following the traditional way of sericulture, silk production is falling day by day. As a result, we gathered information on how to cultivate silkworms in a healthy environment. It is planned to capture photographs of healthy and unwell silkworms and use image processing and a mat lab tool to detect and monitor them. The classification of Healthy and Unhealthy Silkworms is proposed in this paper using Convolutional Neural Networks (CNN). To create the deeper architecture Small kernels are used. The neuron's weight is described as tiny. When compared to all other state-of-the-art methodologies, experimental results demonstrate that CNN archives have a rate of 82.75 percent accuracy with little complexity. Because the larval stage is the worm's newborn stage, environmental elements such as humidity and temperature play a critical impact in its health; the larva's health also influences the quality of the silk produced [1-2]. An automated method is required to monitor these parameters[3-5]. Apart from environmental factors, biological factors such as fungi and bacteria will also have an impact on the worm's health. To address this issue, the system should include a disinfection system, which will allow medicine and other chemicals to be delivered to the worm without causing any harm to the worm's life cycle.

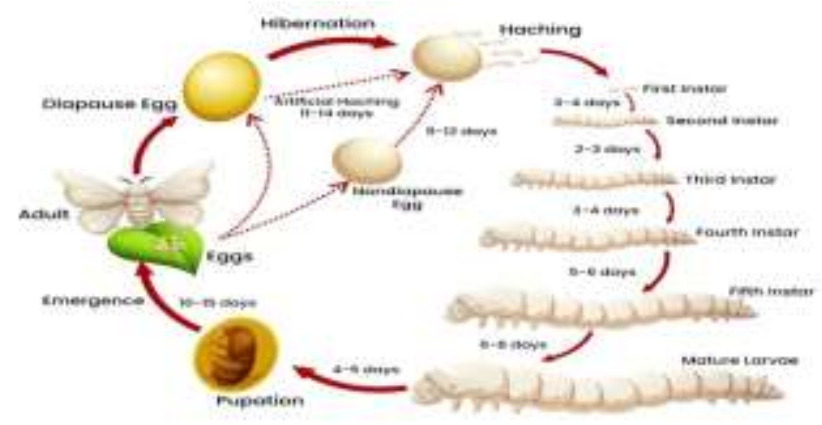

Fig. 1. Metamorphosis of a Cocoon

\section{RELATED WORK}

In the resent days the innovation in the agricultural field is getting revolutionized. The silk farming is one of the tough fields in the agriculture because the requirement of intensive care and more human resources, and the environmental factors also play a major role in the quality and quantity of the silk production. To solve these kinds of problems in other fields dedicated hardware system including sensitive instruments and Image processing techniques are employed [8-9], and system independent software is used to securely connect these hardware units with single or multiple serves[10-11].

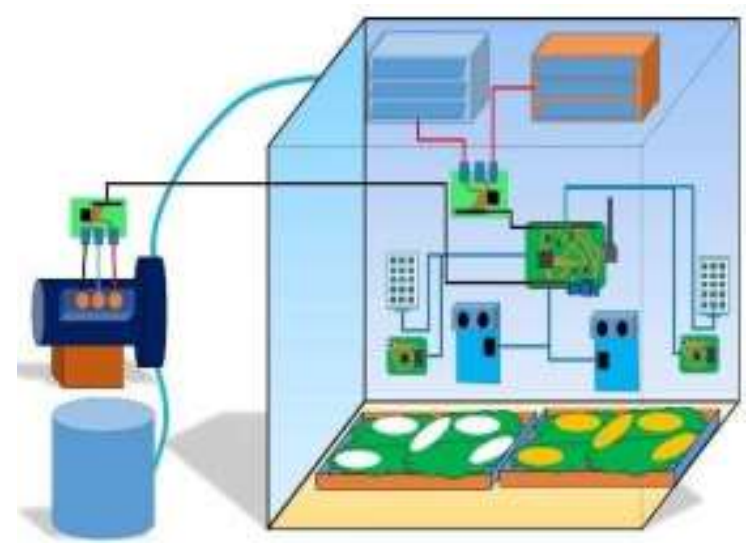

Fig. 2. Smart Implementation of IoT system. 


\section{PROPOSED DESIGN}

In this proposed method, The MATLAB processing is used to detect the silkworm condition. The colour change in the silkworm's body is mostly determined via image processing techniques. The system is built in two parts namely Hardware and software. The system makes use of widely available parts to ensure that parts are always available when they are needed. And employs open-source software such as the Arduino IDE and the Rest API, as well as commercial software such as Matlab for image processing techniques.

\section{A. Hardware}

The system makes use of widely available parts to ensure that parts are always available when they are needed. And employs open-source software such as the Arduino IDE and the Rest API, as well as commercial software such as Matlab for image processing techniques.The Arduino Mega serves as the system's brain, monitoring the system based on data from the humidity sensor, temperature sensor, and soil moisture sensor, and sending that data to the host computer for real-time data logging (see Fig. 3). It also controls electrical devices such as fans, heaters, lights, and spray setups by interacting with the relay module. The camera module is used to monitor the worm's health and quality. The LCD matrix display is used to show the status of various devices and sensors. It is designed in such a way that additional functionality can be added without interfering with the current functionality.

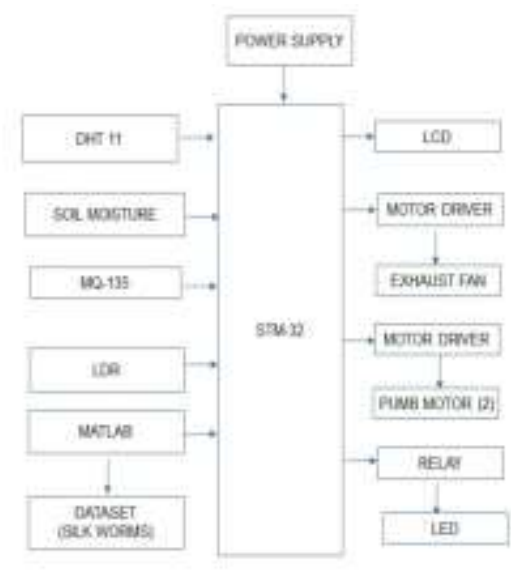

Fig. 3. Block diagram of Hardware

\section{B. Software}

The Arduino IDE is used to create code for the Arduino Mega, which monitors and controls external devices and sensors. Matlab is used for image processing, which aids in recognising and detecting diseased worms and alerting the system (see Fig. 4). Segmentation, edge detection, feature extraction, and noise cancellation are all part of the image processing algorithm. CNN-based classification does not necessitate separate feature extraction steps. CNN is used to calculate the feature value. The image of a healthy and nonhealthy silkworm was classified. As a result, the complexity and computation time are low, while the accuracy is high. Finally, the classification results as healthy and Non-healthy silkworm based on the probability score value.

\section{Image processing}

The process of performing operations on images in order to improve them or extract useful information from them is known as image processing. It is a type of signal processing in which an image is used as input and an image or image characteristics/features are used as output. One of today's fastest growing technologies Image processing is. It is also a core research area in engineering and computer science.

Image processing basically includes the following three steps:

- Importing the image via image acquisition tools;

- Analyzing and manipulating the image;

- Output in which result can be altered image or report that is based on image analysis.

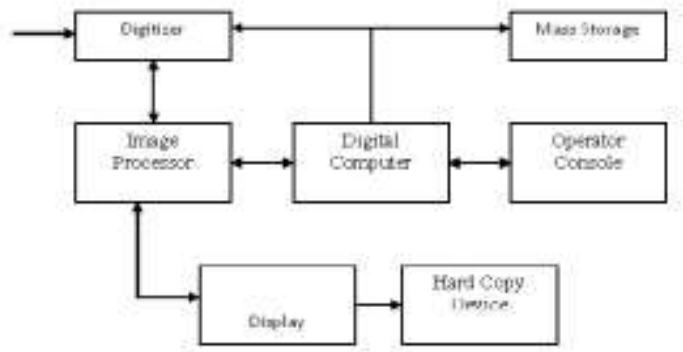

Fig. 4. Flow Chart of Image Processing

Deep learning

Deep learning is a subset of machine learning that teaches computers to do what humans do instinctively: learn from experience. Deep learning is well suited to image recognition, which is critical for solving problems like facial recognition, motion detection, and many advanced driver assistance technologies like lane departure warning. Models are trained using a large set of labelled data and neural network architectures with many layers, typically including some convolutional layers. Training these models is computationally intensive, but it can usually be sped up by using a high-performance GPU. This diagram depicts how convolutional neural networks classify new images by combining layers that automatically learn features from many images.
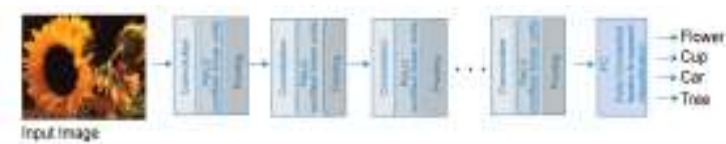

Fig.4. Deep learning working

\section{CONVOLUTIONAL NEURAL NETWORK (CNN)}

A Convolutional Neural Network is the Deep Learning system which will take an input image, assign relevance (learnable weights and biases) to varied aspects/objects within the image, and distinguish between them. The architecture of a ConvNet is analogous thereto of the connectivity pattern of Neurons within the Human Brain and was inspired by the organization of the visual area. Individual neurons answer stimuli only during a restricted region of the field of vision referred to as the Receptive Field. a set of such fields overlap to hide the whole visual cortex . 


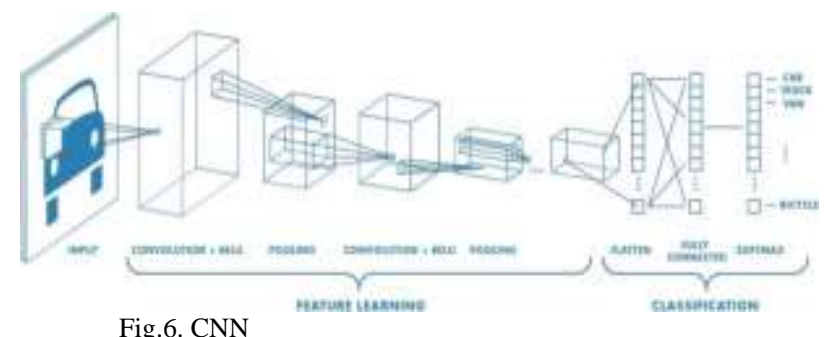

Fig.6. CNN

\section{TYPES OF LAYERS}

- Input layer

- Convolutional layer

Convolutional layers are the layers where filters are applied to the first image, or to other feature maps during a deep CNN. this is the most often where most of the user-specified parameters are within the network. the foremost important parameters are the amount of kernels and therefore the size of the kernels.

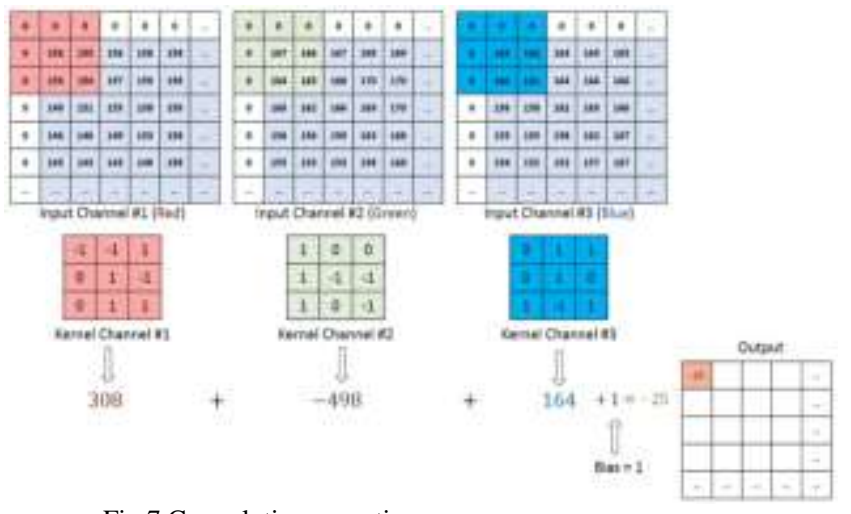

Fig.7.Convolution operation

- Maxpooling layer

Max Pooling returns the utmost value from the portion of the image covered by the Kernel. Max Pooling also performs as a Noise Suppressant. It discards the noisy activations altogether and also performs de-noising along side dimensionality reduction.

max pooling

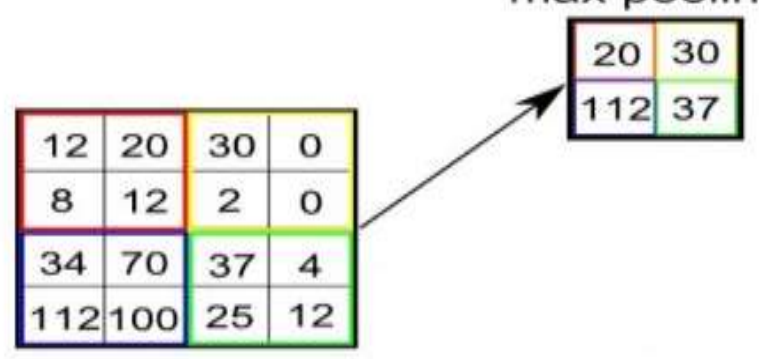

Fig.8.Maxpooling

- Relu layer

The ReLU layer applies the function to all or any of the values within the input volume. this layer just changes all the negative values to 0 .

- Batch normalization layer

Batch normalisation is a layer that enables each layer of the network to perform learning independently. It is used to normalise the previous layers' output. In normalisation, the

Volume 24, Issue 1, January - 2022
- Soft max layer

Softmax is useful because it converts the output of your neural network's last layer into what is essentially a probability distribution.

- Fully connected layer

Adding a Fully-Connected layer is a (usually) low-cost method of learning non-linear combinations of the high-level features represented by the convolutional layer's output. In that space, the Fully-Connected layer is learning the possibly non-linear function.
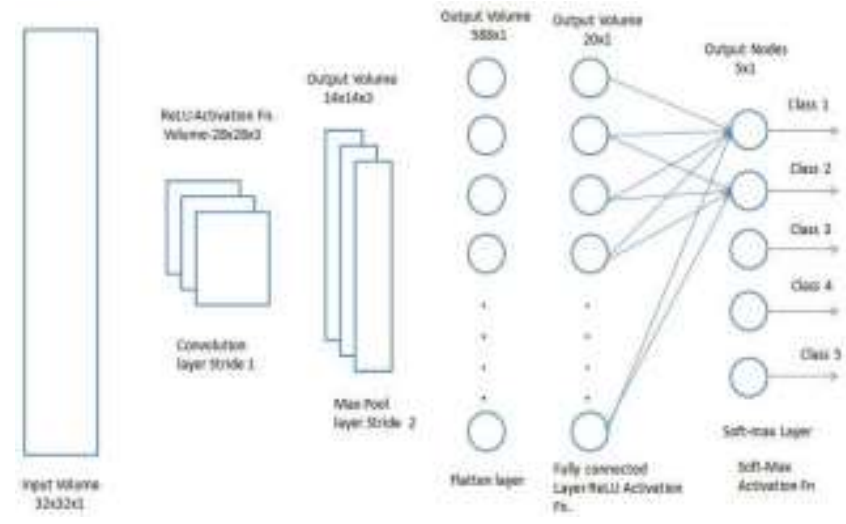

Fig.9.Fully connected layer

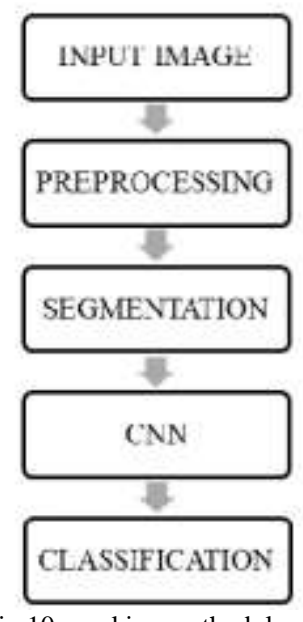

Fig.10. working methodology

\section{MATLAB}

MATLAB is a numerical computing environment and the programming language is fourth-generation. MATLAB, created by Math Works, allows for matrix manipulations, function and data plotting, algorithm implementation, user interface creation, and interfacing with programmes written in other languages such as $\mathrm{C}, \mathrm{C}++$, Java, and Fortran.

\section{RESULT}

The fully built system see Fig.11 can monitor environmental factors such as temperature, temperature and rain using the sensors, it also controls the culture environment using fans and heaters. The image processing algorithms employed can successfully resize the image by edge detectiton and maxpooling as shown in Fig.12, the processed images enable to differentiate the healthy and unhealthy silkworms and detects the diseased silkwormsee Fig. 13. The LCD matrix successfully displayed all sensors live status without fail as shown in Fig.14. the Fig.15 shows accuracy of the trained system. 


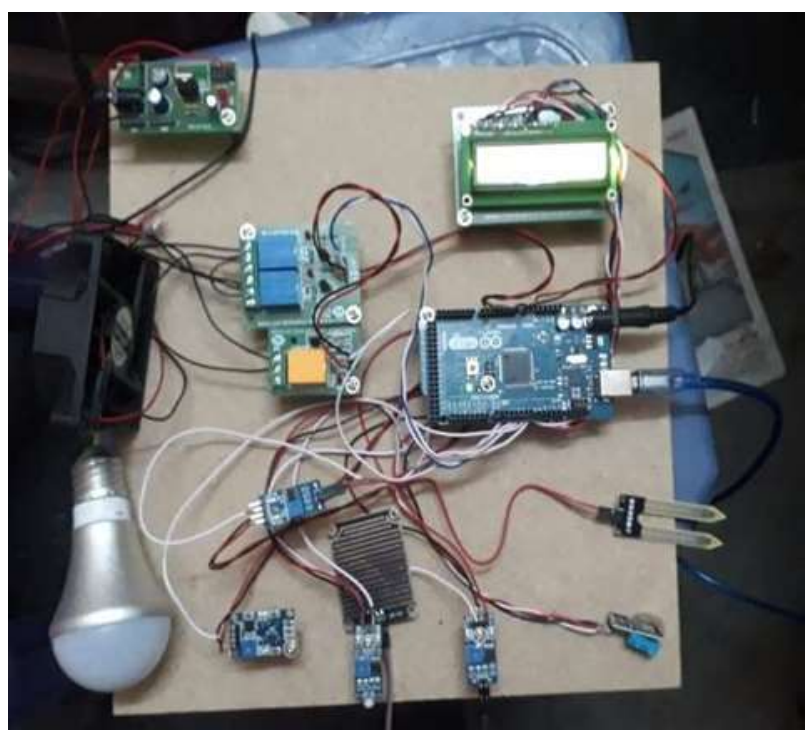

Fig. 11. Implementaion of smart Sericulture

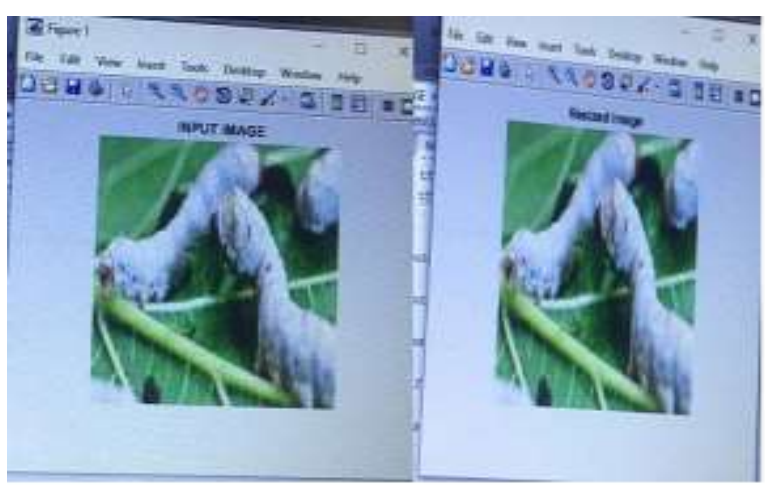

Fig. 12. Worm resized images
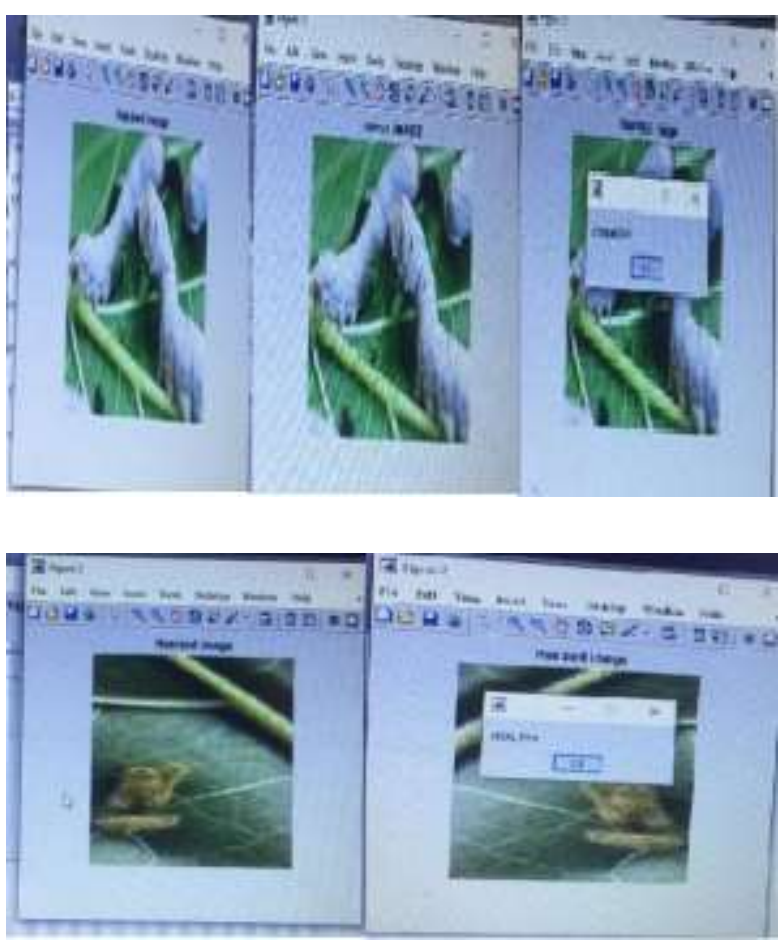

Fig.13. output the healthy and unhealthy silkworms are detected
SFART SER ICULTUF PROTECT

TEFIF:

LAFD WIET
PLIPIF DFF

LAHD DFY
FLIFP DIT

NIGHT UISIDH BULB 마

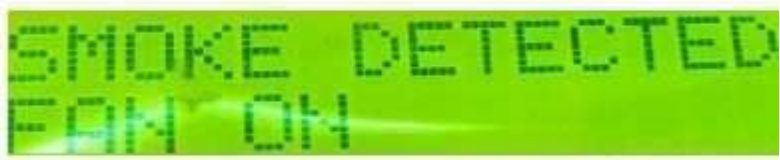

Fig. 14. The Different Status Of Sensores Worm Images
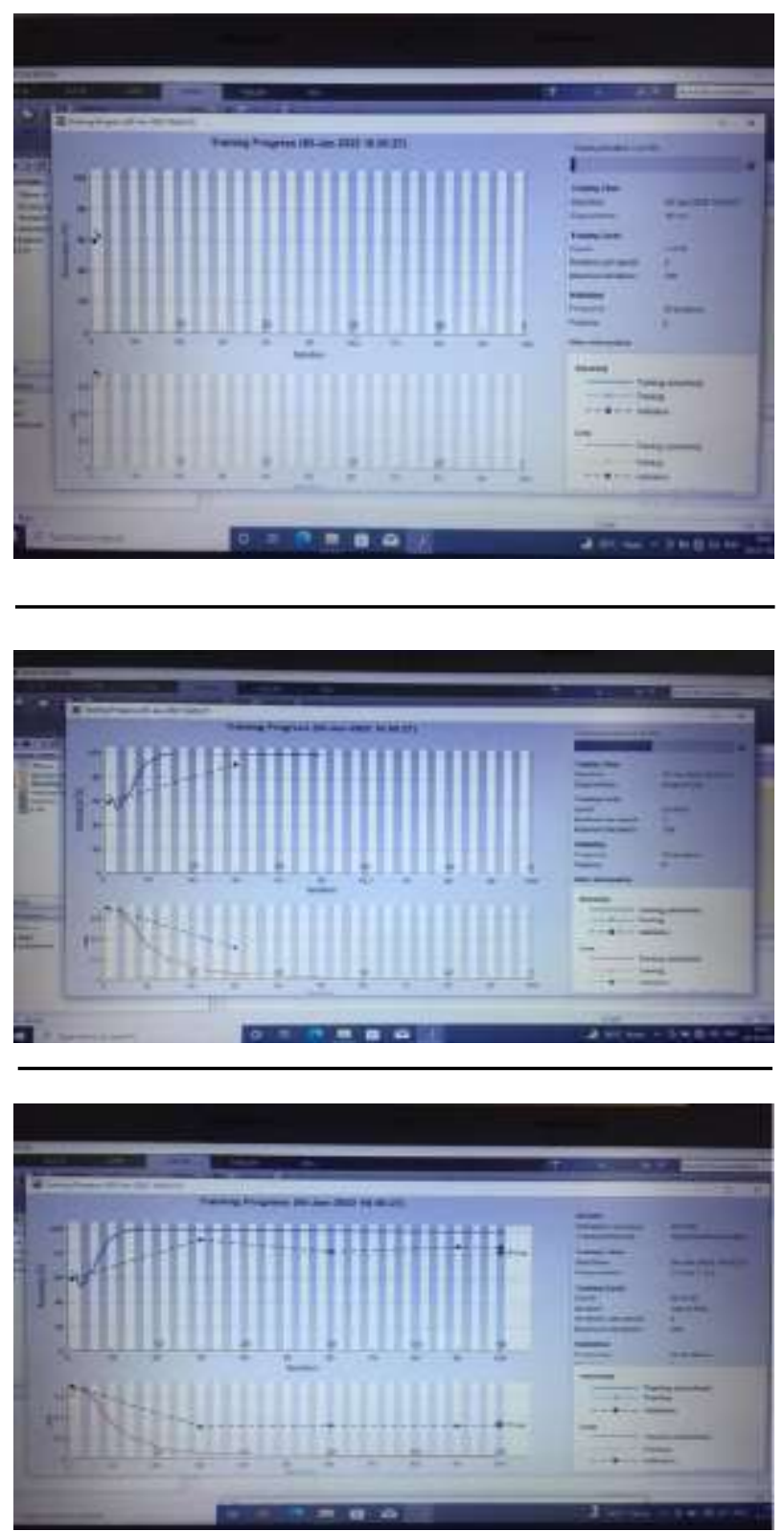

Fig.15.System training accuracy

Volume 24, Issue 1, January - 2022 


\section{CONCLUSION}

The system can be used to improve the quality of silk farm output with very less human interventions. In addition to quality controlling and monitoring the system can be programmed to have extended functions in the silk farm as required by the farmer. The main goal of this research work is to design efficient healthy and unhealthy silkworm classification with high accuracy, performance and low complexity. A convolution neural network-based Classification is also introduced in the proposed scheme to improve accuracy and reduce computation time. Also the classification results are given as unhealthy or healthy silkworm. CNN is a deep learning technique. As a result, only the final layer is trained. CNN also extracts raw pixel values with depth, width, and height feature values. Finally, to achieve high accuracy, the Gradient decentbased loss function is used. The training and validation accuracy, as well as the validation loss, are computed.

\section{REFERENCE}

[1] V.K.RAHMATHULLA, "MANAGEMENT OF CLIMATIC FACTORS

FORSUCCESSFUL SILKWORM (BOMBYXMORI) CROP AND HI GHER SILKPRODUCTION: A REVIEW", HINDAWI PUBLISHING CORPORATION PSYCHE, VOLUME 2012, ARTICLE ID 121234 12 PAGESDOI: $10.1155 / 2012 / 121234$.

[2] MUBASHARHUSSAIN, SHAKIL AHMAD KHAN, MUHAMMAD NAEEM ANDM. FAROOQNASIR, "EFFECT OF REARING TEMPE
-RATURE AND HUMIDITY ONFECUNDITY AND FERTILITY OF SILKWORM, BOMBYXMORI L. (LEPIDOPTERA: BOMBYCIDAE)" PAKISTAN J. ZOOL., VOL. 43(5), PP. 979-985, 2011.

[3] KONSTANTINOS N. PLATANIOTIS, DIMITRIOSANDROUTSOS, SRI VIN AYAGAMOORTHY, AND ANASTASIOS N. VENETSANOPOULOS, "COLORIMAGE PROCESSING USING ADAPTIVE MULTICHANNEL FILTERS", IEEETRANSACTIONS ON IMAGE PROCESSING, VOL. 6, NO. 7, JULY 1997.

[4] RICARDO L. DE QUEIROZ, "PROCESSING JPEG-COMPRESSED IMAGESAND DOCUMENTS", IEEE TRANSACTIONS ON IMAGE PROCESSING, VOL. 7, NO. 12, DECEMBER 1998.

[5] AN EFFICIENT CLASSIFICATION OF MRI BRAIN IMAGES BY MUHAMMAD ASSAM , HIRA KANWAL, UMAR FAROOQ, SAID KHALID SHAH , ARIF MEHMOOD , AND GYU SANG CHOI

[6] M.A. DIXIT ET AL, "INTELLIGENT CONTROL SYSTEM FOR SERICULTURE", 2015 INTERNATIONAL CONFERENCE ON PERVASIVE COMPUTING (ICPC).

[7] DIVYA DARSHINI.B, ADARSH.B.U, SHIVAYOGAPPA.H.J, NAVYA.K.N, "AUTOMATED SMART SERICULTURE SYSTEM BASED ON 6LOWPAN AND IMAGE PROCESSING TECHNIQUE" 2016 INTERNATIONAL CONFERENCE ON COMPUTER COMMUNICATION AND INFORMATICS (ICCCI -2016), JAN. 07 09, 2016, COIMBATORE, INDIA

[8] CHETAN DWARKANI M ET AL, "SMART FARMING SYSTEM USING SENSORS FOR AGRICULTURAL TASK AUTOMATION", 2015 IEEE INTERNATIONAL CONFERENCE ON TECHNOLOGICAL INNOVATIONS IN ICT FOR AGRICULTURE AND RURAL DEVELOPMENT (TIAR 2015) 